\title{
Low-loss grating for coupling to a high-finesse cavity
}

\author{
A. Bunkowski, O. Burmeister, P. Beyersdorf, K. Danzmann, and R. Schnabel \\ Max-Planck-Institut für Gravitationsphysik (Albert-Einstein-Institut), and Institut für Atom- und Molekülphysik, \\ Universität Hannover, Callinstrasse 38, 30167 Hannover, Germany
}

T. Clausnitzer, E.-B. Kley, and A. Tünnermann

Institut für Angewandte Physik, Friedrich-Schiller-Universität Jena, Max-Wien-Platz 1, 07743 Jena, Germany

Received June 24, 2004

\begin{abstract}
A concept for a low-loss all-reflective cavity coupler is experimentally demonstrated at a wavelength of $1064 \mathrm{~nm}$. A $1450-\mathrm{nm}$ period dielectric reflection grating with a diffraction efficiency of $0.58 \%$ in the -1 st order is used in the 2nd-order Littrow configuration as a coupler to form a cavity with a finesse of 400. The application of such reflective low-loss cavity couplers in future generations of gravitational-wave detectors and implementation issues are discussed. (C) 2004 Optical Society of America
\end{abstract}

OCIS codes: $\quad 050.1950,120.3180,230.1360$.

An international network of first-generation, kilometer-scale, earthbound laser-interferometric gravitational-wave (GW) detectors, consisting of the Laser Interferometer Gravitational Wave Observatory (LIGO), ${ }^{1,2}$ the GEO $600,{ }^{2}$ the TAMA $300,{ }^{3}$ and the VIRGO project, ${ }^{4}$ is currently moving from the commissioning phase to the long-term data-taking operational phase. These detectors are Michelson interferometers. Power recycling and arm cavities are two techniques being used to increase the laser power in the interferometer and hence the detector sensitivity. Both techniques utilize cavities to which laser light is coupled via a partially transmitting mirror. For first-generation detectors the light power inside the interferometer will be of the order of $10 \mathrm{~kW}$ at a wavelength of $1064 \mathrm{~nm}$. To increase the detection sensitivity even further, future GW interferometers will use light power of the order of megawatts for which heating effects in the transmissive elements become an issue. Power absorption in the substrates leads to thermal lensing and also to deformation of the optical surface. These distortions will limit the circulating power below the level that is necessary to optimize quantum noise. To reduce thermal noise, cryogenic techniques for the main optics are likely to be used in third-generation GW detectors. Absorbed heat in the substrates will worsen the cooling efforts of the optical elements. To avoid heating in the substrate, reflective-grating beam splitters can be used instead of partially transmissive mirrors and beam splitters. ${ }^{5}$ An additional advantage of all-reflective optics within GW detectors is the elimination of the constraint that the substrate materials be optically transparent. Considering opaque substrate materials with superior mechanical properties allows one to lower the thermal noise in the detector.

In proof-of-principle experiments Sun and Byer ${ }^{6}$ demonstrated Michelson and Sagnac interferometers based on all-reflective elements. They also demonstrated a Fabry-Perot coupler concept that is based on high-diffraction-efficiency gratings in the 1st-order Littrow configuration. Drever pointed out that low-diffraction-efficiency gratings could also be used as cavity couplers and argued that the overall losses should be lower than in high-diffractionefficiency elements. An all-reflective interferometer configuration that avoids the use of a 50/50 beam splitter and uses low-diffraction-efficiency gratings and mirrors as the only major optical elements, as depicted schematically in Fig. 1, was proposed. ${ }^{5}$ But to our knowledge no experimental realization of interferometers utilizing low-diffraction gratings has been reported so far. In this Letter we report on the design of a low-loss diffraction grating with a diffraction efficiency of less than $1 \%$ and on the experimental realization of a high-finesse linear cavity that uses the grating as a coupler.

For a laser beam of wavelength $\lambda$ that is incident on a reflection grating the output angle $\Theta_{m}$ of the $m$ th diffracted order is given by the well-known grating equation

$$
d\left(\sin \Theta_{m}-\sin \Theta_{\text {in }}\right)=m \lambda,
$$

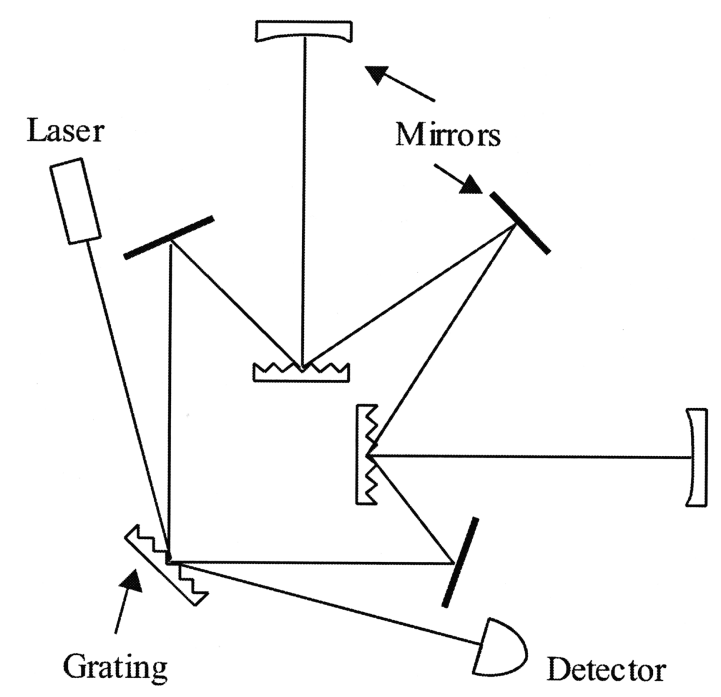

Fig. 1. Interferometer setup proposed by Drever. ${ }^{5}$ 
where $\Theta_{\text {in }}$ is the incidence angle and $d$ is the grating period. If it is used in the 1st-order Littrow configuration ( $\left.\sin \Theta_{\text {in }}=\lambda / 2 d\right)$, a reflection grating can be used as a cavity coupler ${ }^{6}$; the reflected (0th-order) beam is used to couple light into the resonator. The finesse of such a resonator is limited by the maximum diffraction efficiency of the 1st order of the grating. However, if it is used in the 2nd-order Littrow configuration, as shown in Fig. 2, the diffracted (-1st) order is used to couple the cavity. Then, the maximum reflectivity $R_{0}$ of the grating under normal incidence is the limiting factor for the finesse of the cavity.

Standard coating techniques can routinely produce multilayers with a reflectivity greater than $99.98 \%$. This value is to be compared with routinely produced maximum diffraction efficiencies of $\sim 95 \%$. $^{7}$ To our knowledge the highest value ever reported so far is also no greater than $99 \% .8$ Therefore the 2nd-order Littrow configuration is the appropriate choice for efficient low-loss coupling to a linear resonator.

Every diffraction order that is allowed by the grating equation will contain some light power. To reduce overall losses in the device one should choose the grating period $d$ so that only the diffraction orders that are going to be used are allowed by the grating equation. Only the \pm 1 orders for normal incidence are needed in our case, which suggests that

$$
\lambda<d<2 \lambda \text {. }
$$

A common way of manufacturing high-efficiency dielectric reflection gratings is to etch a periodic structure into the top layer of a dielectric multilayer stack as is done for gratings used in high-power chirped-pulse amplification. ${ }^{9}$ For the low diffraction efficiency grating needed for our application we used a different approach. We first etched the grating into a substrate and then overcoated it so that the dielectric layers effectively formed a volume grating, as can be seen from Fig. 3. A shallow binary structure with a depth of $40-50 \mathrm{~nm}$, a ridge width of $840 \mathrm{~nm}$, and a period of $d=1450 \mathrm{~nm}$ was generated by electron-beam lithography and reactive-ion beam etching on top of a fusedsilica substrate. The applied multilayer stack was composed of 32 alternating layers of silica $\left(\mathrm{SiO}_{2}\right)$ and tantalum pentoxide $\left(\mathrm{Ta}_{2} \mathrm{O}_{5}\right)$. The diffraction efficiency of 1064-nm light with a polarization plane parallel to the grating grooves and perpendicular to the plane of incidence ( $s$ polarization) was measured to be $\eta=(0.58 \pm 0.04 \%)$.

Figure 4 shows the experimental setup of the allreflective Fabry-Perot cavity. A highly reflective mirror with a radius of curvature of $1.5 \mathrm{~m}$ mounted on a piezoelectric transducer to allow for cavity-length control was placed parallel to the grating surface at a distance of $43 \mathrm{~cm}$. An $s$-polarized beam of $50 \mathrm{~mW}$ from a 1.3-W, 1064-nm diode-pumped Nd:YAG laser was used. The angle of incidence corresponded to 2ndorder Littrow configuration $\Theta_{\text {in }}=\arcsin (\lambda / d) \approx 47.2^{\circ}$. The circulating and the reflected power from the cavity were observed by monitoring the leakage from the high reflector and from the 0th-order of the grating, respectively.
High circulating powers inside the cavity also demand good mode matching of the input beam and the cavity mode. Note that our grating couples modes at different angles of incidence, which changes the ratio of horizontal and vertical mode widths. The following relation holds for the horizontal width $w_{h}$ of the beam:

$$
w_{\text {in }, h} / w_{m, h}=\cos \Theta_{\text {in }} / \cos \Theta_{m},
$$

where in and $m$ refer to the incoming and the diffracted beam, respectively. For our setup an input
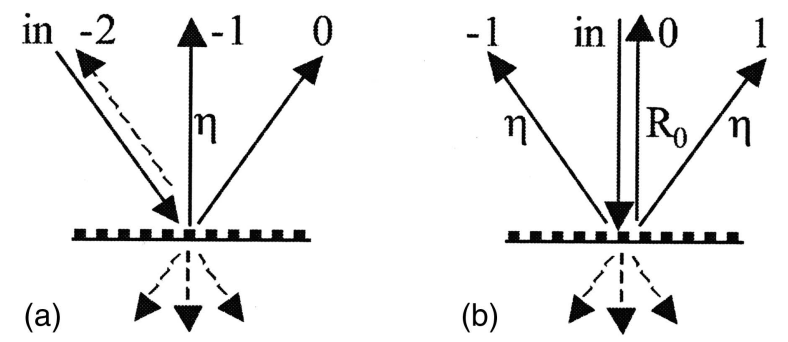

Fig. 2. Reflected and transmitted orders from a reflection grating in the 2nd-order Littrow configuration: (a) incident beam at the Littrow angle, (b) normal incidence.

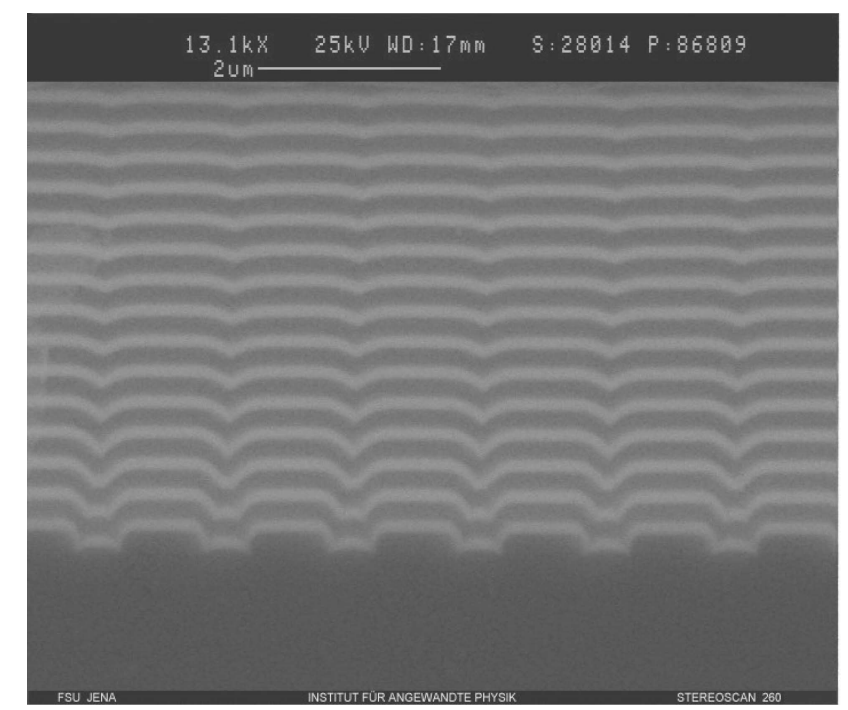

Fig. 3. Cross section of an overcoated binary grating (scanning electron microscope image).

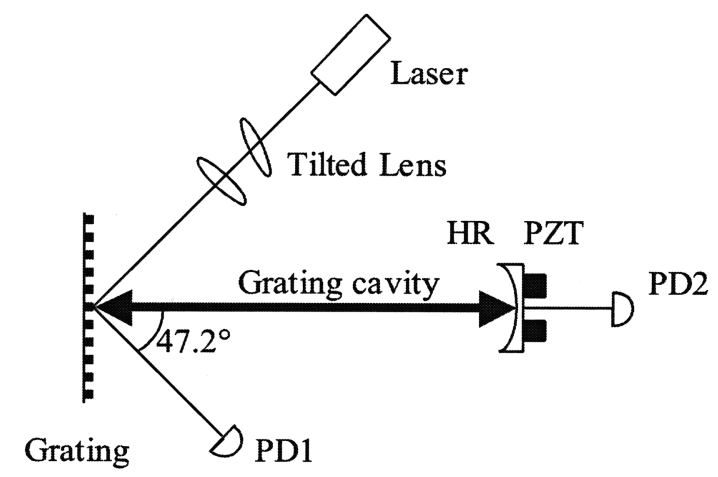

Fig. 4. Experimental setup of the demonstrated grating Fabry-Perot cavity: HR, high reflector; PZT, piezoelectric transducer; PD1, PD2, photodiodes. 


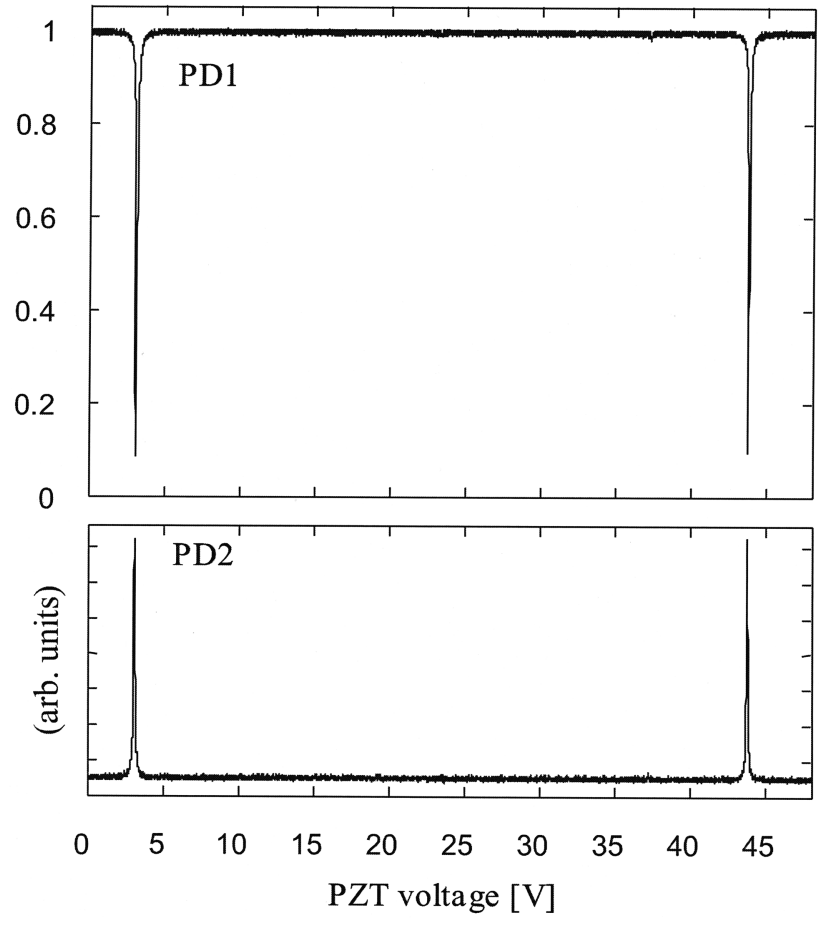

Fig. 5. Measured signals of the two photodiodes according to Fig. 4 versus the piezoelectric transducer ramp voltage over one free spectral range. PD1, reflected power normalized to the power of the incident beam; PD2, circulating power in the cavity.

beam with an elliptical profile having a horizontal width of 1.47 times $\left(w_{h} / w_{v}=\cos \Theta_{m} / \cos \Theta_{\text {in }} \approx 1.47\right)$ the vertical width produced the desired round beam profile in the diffracted beam. The profile was generated by a pair of lenses from which one lens was tilted horizontally to have different focal lengths for the $v$ and $h$ directions.

Figure 5 shows the transmission and reflection interference fringes for the cavity with a measured cavity finesse of $400 \pm 20$. More than $99 \%$ of the power was measured to be in the $\mathrm{TEM}_{00}$ mode, indicating excellent mode matching. With the measured value of the finesse and the known reflectance of the highreflection mirror, one can calculate the overall losses $A$, which are defined by $A=1-R_{0}-2 \eta$, where $R_{0}$ is the reflectance for normal incidence. We find that $A=(0.38 \pm 0.2) \%$. Losses are due to transmitted orders and scattered light.

The partially transmissive mirrors conventionally used for coupling to a linear Fabry-Perot cavity can be considered two-port devices with simple phase relations between the two ports. The input coupler introduced here, however, is a three-port device. Light entering one port will always couple to all three ports. The phase relations of the light in the three different ports are more complex than in a two-port device. They depend on the diffraction efficiencies of the different orders and can be calculated with a scattering matrix formalism. ${ }^{10}$ Because of the additional port, new GW detector topologies can be obtained. The scheme shown in Fig. 1, for example, uses two linear grating cavities in the arms. On resonance these cavities will retroreflect most of the power incident on the grating, while the signal sidebands generated in the arms will be split equally between the two output ports of the cavity. Therefore the power and the signal are taking different paths in the interferometer. A detailed analysis of the phases of the three ports of the coupler as well as their effects on the properties of the proposed interferometer is in preparation and will be presented in the future.

In conclusion, we have experimentally demonstrated that a low-efficiency grating can be used as a cavity input coupler with low loss. A cavity with a finesse of 400 was constructed that far exceeded the best finesse values for an all-reflective cavity that had been previously reported. ${ }^{6}$ We plan to optimize the design and manufacturing process of the gratings to produce gratings with even lower diffraction efficiency and overall losses. These gratings will have high potential to be implemented in future GW detector configurations.

This research was supported by the Deutsche Forschungsgemeinschaft within the Sonderforschungsbereich TR7. A. Bunkowski's e-mail address is alexander.bunkowski@aei.mpg.de.

\section{References}

1. A. Abramovici, W. E. Althouse, R. W. P. Drever, Y. Gürsel, S. Kawamura, F. J. Raab, D. Shoemaker, L. Sievers, R. E. Spero, K. S. Thorne, R. E. Vogt, R. Weiss, S. E. Whitcomb, and M. E. Zucker, Science 256, 325 (1992).

2. The LIGO Scientific Collaboration, Nucl. Instrum. Methods Phys. Res. A 517, 154 (2004).

3. The TAMA Collaboration, Phys. Rev. Lett. 86, 3950 (2001).

4. The VIRGO Collaboration, Class. Quantum Grav. 21, 709 (2004).

5. R. W. P. Drever, in Proceedings of the Seventh Marcel Grossman Meeting on General Relativity, M. Keiser and R. T. Jantzen, eds. (World Scientific, Singapore, 1995).

6. K.-X. Sun and R. L. Byer, Opt. Lett. 23, 567 (1997).

7. T. Clausnitzer, J. Limpert, K. Zöllner, H. Zellmer, H.-J. Fuchs, E.-B. Kley, A. Tünnermann, M. Jupé, and D. Ristau, Appl. Opt. 42, 6934 (2003).

8. J. A. Britten, S. J. Bryan, L. J. Summers, H. T. Nguyen, B. W. Shore, and O. Lyngnes, in Conference on Lasers and Electro-Optics, Vol. 73 of OSA Trends in Optics and Photonics Series (Optical Society of America, Washington, D.C., 2002), pp. CPDB7-1-4.

9. B. W. Shore, M. D. Perry, J. A. Britten, R. D. Boyd, M. D. Feit, H. T. Nguyen, R. Chow, G. E. Loomis, and L. Li, J. Opt. Soc. Am. A 14, 1124 (1997).

10. A. Siegman, Lasers (University Science, Mill Valley, Calif., 1986). 